\title{
BMJ Open Comparison of olanexidine versus povidone-iodine for preventing surgical site infection in gastrointestinal surgery: study protocol for a multicentre, single- blind, randomised controlled clinical trial
}

Masashi Takeuchi, ${ }^{1}$ Hideaki Obara, ${ }^{1}$ Hirofumi Kawakubo, ${ }^{1}$ Masahiro Shinoda, ${ }^{1}$ Koji Okabayashi, ${ }^{1}$ Shuhei Mayanagi, ${ }^{1}$ Tomoyuki Irino, ${ }^{1}$ Kazumasa Fukuda, ${ }^{1}$ Rieko Nakamura, ${ }^{1}$ Norihito Wada, ${ }^{1}$ Minoru Kitago, ${ }^{1}$ Hiroshi Yagi, ${ }^{1}$ Yuta Abe,${ }^{1}$ Go Oshima, ${ }^{1}$ Shutaro Hori, ${ }^{1}$ Masashi Tsuruta, ${ }^{1}$ Takashi Ishida, ${ }^{1}$ Takahiro Yokose, ${ }^{1}$ Kazuya Hirukawa, ${ }^{1}$ Yoh Isobe, ${ }^{2}$ Yasuhito Sekimoto, ${ }^{2}$ Hirohisa Harada, ${ }^{3}$ Yusuke Maeda, ${ }^{3}$ Masaya Shito, ${ }^{4}$ Takayuki Kondo, ${ }^{4}$ Yasunori Sato, ${ }^{5}$ Yuko Kitagawa ${ }^{1}$

To cite: Takeuchi M, Obara $\mathrm{H}$, Kawakubo $\mathrm{H}$, et al. Comparison of olanexidine versus povidoneiodine for preventing surgical site infection in gastrointestinal surgery: study protocol for a multicentre, single-blind, randomised controlled clinical trial. BMJ Open 2019;9:e028269. doi:10.1136/ bmjopen-2018-028269

\section{- Prepublication history and} additional material for this paper are available online. To view these files, please visit the journal online (http://dx.doi. org/10.1136/bmjopen-2018028269).

Received 29 November 2018 Revised 14 March 2019 Accepted 1 May 2019

Check for updates

(C) Author(s) (or their employer(s)) 2019. Re-use permitted under CC BY-NC. No commercial re-use. See rights and permissions. Published by BMJ.

For numbered affiliations see end of article.

Correspondence to

Dr Hideaki Obara;

obara.z3@keio.jp

\section{ABSTRACT}

Introduction The prevalence of surgical site infection (SSI) remains higher in gastrointestinal surgery than in other surgeries. Although several guidelines have indicated the efficacy of chlorhexidine and povidone-iodine in reducing the SSI rate, the optimal recommendation has still not been established. Therefore, it is necessary to determine the more effective antiseptic for surgical site preparation. Olanexidine (1.5\% olanedine, Otsuka Pharmaceutical Factory, Tokushima, Japan), which is a new antiseptic in Japan, has antimicrobial activity against a wide range of bacteria, including Gram-positive and Gram-negative bacteria. Our study will contribute to determining a new antiseptic for use in gastrointestinal and other surgeries.

Methods and analysis We propose a multicentre, randomised controlled clinical trial for comparing two treatments, that is, $1.5 \%$ olanexidine or $10 \%$ povidoneiodine, for surgical skin preparation to prevent SSI in clean-contaminated gastrointestinal surgeries with surgical wounds. Patients aged $\geq 20$ years at the time of consent will be included. The primary outcome measure is the 30-day postoperative SSI rate. For the primary analysis, which is aimed at comparing the treatment effects, the adjusted risk ratio and its $95 \% \mathrm{Cl}$ will be estimated using the Mantel-Haenszel method.

Ethics and dissemination The protocol was first approved by the Institutional Review Board of Keio University School of Medicine, followed by the institutional review board of each participating site. Participant recruitment began in June 2018. The final results will be published in international peer-reviewed medical journals.

Trial registration number UMIN 000031560; Preresults.

\section{Strengths and limitations of this study}

This is the first study to evaluate the effect of olanexidine, which has been commercially available in Japan since 2015.

- The study design is a multicentre, single-blind, randomised controlled clinical trial.

- The primary outcome measure is the 30 -day postoperative surgical site infection rate.

- This study is limited to a Japanese population, which could introduce an element of selection bias, because olanexidine is only commercially available in Japan.

\section{INTRODUCTION}

Surgical site infection (SSI) is one of the most common nosocomial infections in surgical patients. ${ }^{1}$ The rate of SSI in gastrointestinal surgery, in particular, is higher than in other surgeries, such as cardiothoracic surgery, gynaecological surgery and neurosurgery, ${ }^{2-4}$ and it has been reported that $10 \%$ to $30 \%$ of patients suffer from SSI after gastrointestinal surgery. ${ }^{5}$ SSI causes prolonged hospitalisation and delay of postoperative therapy and increased medical costs of \$1300-5000 per person for inpatient treatment, including antibiotic therapy. ${ }^{6}$ Therefore, prevention of SSI is extremely important for both the patient and all medical practitioners involved in the surgery.

Many perioperative measures for decreasing SSI have been reported, including enhanced 
nutritional support, perioperative oxygenation, surgical technique, wound dressing and use of an antimicrobial agent. ${ }^{18}$ Surgical site preparation is useful for preventing SSI because it can remove micro-organisms from the skin. Thus far, two types of preparations, povidone-iodine and chlorhexidine-alcohol, ${ }^{9-12}$ have been commonly used as preoperative antiseptic procedures worldwide. The Centers for Disease Control and Prevention (CDC) guideline only recommends skin preparation with an alcohol-containing agent if there are no contraindications to its use, and other guidelines do not favour one antiseptic agent over another for skin preparation. ${ }^{13} 14$ Both preparations have broad-spectrum antibacterial effectiveness; however, povidone-iodine's activity is known to decrease in the presence of organic materials including blood or pus. ${ }^{15}$ In contrast, chlorhexidine-alcohol has high antibacterial activity against some pathogens, such as methicillin-resistant Staphylococcus aureus (MRSA) ${ }^{15}$ and vancomycin-resistant enterococci (VRE); nevertheless, it is associated with inflammability, is more expensive than povidone-iodine and has been linked to allergic reactions. ${ }^{1617}$ Therefore, it is necessary to determine the more effective antiseptic for surgical site preparation.

Olanexidine (1.5\% Olanedine; Otsuka Pharmaceutical Factory, Tokushima, Japan), which is a new antiseptic, is one of the biguanide bactericidal disinfectants that contains olanexidine gluconate as its active ingredient. ${ }^{18} 19$ It has been commercially available in Japan since 2015. It can disrupt membrane integrity by binding to the cell membrane, resulting in irreversible leakage of intracellular components, which is the mechanism underlying its bactericidal and fungicidal activities. ${ }^{18}$ Olanexidine has antimicrobial activity against a wide range of bacteria, including Gram-positive and Gram-negative bacteria. Moreover, Inoue et al reported that compared with chlorhexidine-alcohol and povidone-iodine, olanexidine showed more potent bactericidal activity against MRSA and VRE both in vitro and in vivo. ${ }^{18}$ Therefore, the use of olanexidine is highly expected to lead to decreases in the SSI rate. However, to date, no study has evaluated the effectiveness and safety of olanexidine compared with conventional antiseptics in large-scale clinical trials.

In this multicentre, randomised controlled clinical trial, we aim to evaluate whether olanexidine or povidone-iodine, which is the conventional skin antiseptic used in Japan, is useful for preventing SSI in gastrointestinal surgery. We hypothesise that olanexidine will be more useful for preventing SSI than povidone-iodine without increasing toxicity.

\section{METHODS}

\section{Trial design}

This is a multicentre, prospective, randomised, blinded-endpoint trial designed to assess the efficacy of $1.5 \%$ olanexidine for surgical skin preparation for preventing SSIs in gastrointestinal surgery. The trial was designed and will be independently conducted by Keio University
Table 1 Definition of the wound classes

\begin{tabular}{|c|c|}
\hline Wound class & Definition \\
\hline Class I (clean) & $\begin{array}{l}\text { An uninfected operative wound in which } \\
\text { no inflammation is encountered and the } \\
\text { respiratory, alimentary, genital or uninfected } \\
\text { urinary tracts are not entered. }\end{array}$ \\
\hline $\begin{array}{l}\text { Class II (clean- } \\
\text { contaminated) }\end{array}$ & $\begin{array}{l}\text { Operative wounds in which the respiratory, } \\
\text { alimentary, genital or urinary tracts are } \\
\text { entered under controlled conditions and } \\
\text { without unusual contamination. }\end{array}$ \\
\hline $\begin{array}{l}\text { Class III } \\
\text { (contaminated) }\end{array}$ & $\begin{array}{l}\text { Includes open, fresh and accidental } \\
\text { wounds. }\end{array}$ \\
\hline $\begin{array}{l}\text { Class IV (dirty- } \\
\text { contaminated) }\end{array}$ & $\begin{array}{l}\text { Includes old traumatic wounds with } \\
\text { retained devitalised tissue and those } \\
\text { that involve existing clinical infection or } \\
\text { perforated viscera. }\end{array}$ \\
\hline
\end{tabular}

with approval from the ethics committee of Keio University School of Medicine in accordance with the principals of the Declaration of Helsinki. All analyses will be conducted by Keio University, independent of the sponsor, according to the prespecified statistical analysis plan (SAP). As a prospective randomised controlled trial, the study strategy will be constructed and presented in accordance with the recommendations of the Standard Protocol Items: Recommendations for Interventional Trials statement.

\section{Eligibility criteria}

Eligible patients are those who meet all the following inclusion criteria and who do not have any listed exclusion criteria.

Inclusion criteria

1. Scheduled to undergo elective gastrointestinal surgery involving the oesophagus, stomach, duodenum, small intestine, colorectal, liver, biliary tract and pancreas that has a class II surgical wound (table 1).

2. Age $\geq 20$ years at the time of consent by non-blinded investigators.

3. Provision of written informed consent by the patient.

Exclusion criteria

1. Allergy to olanexidine gluconate or povidone-iodine.

2. Unable to undergo follow-up 30 days postoperatively.

3. Active bacterial infection at the time of informed consent (except for viral hepatitis).

4. Antimicrobial therapy on the day before surgery.

5. Undergoing non-elective surgery or surgery requiring antisepsis of the mucosal surfaces or surgical wound sites.

6. Unsuitable conditions for safe conduct of this trial according to the non-blinded investigators.

\section{Intervention}

Study arm A (experimental group): Surgical skin antisepsis with an aqueous formulation of $1.5 \%$ olanexidine is administered immediately before gastrointestinal surgery. 
Study arm B (control group): Surgical skin antisepsis with an aqueous formulation of $10 \%$ povidone-iodine is administered immediately before gastrointestinal surgery.

\section{Treatment protocol}

The antisepsis should be applied widely in consideration of the drain site and length of the skin incision. We apply agents from the papilla (in cases of oesophageal surgery; the neck) with a cranial limit and to the upper thigh with a caudal limit. The duration of application of both antiseptics is at least $1 \mathrm{~min}$. After waiting $3 \mathrm{~min}$ to allow the antiseptics to dry, the operation is started. Olanexidine is administered by ready-to-use applicators. One olanexidine applicator will be used in surgery; however, if surgeons determine that disinfection is inadequate, an additional applicator can be added. Povidone-iodine is administered by a brush or by compression using pliers.

We used other measures to prevent SSI in our protocol as follows:

1. Administering standard antibiotic prophylaxis before making the surgical incision.

2. Using absorbable sutures for wound closure and recommending the use of antimicrobial-coated sutures.

3. Recommending the use of a wound protector (the types used are the Alexis wound protector (Medical Leaders Co., Tokyo, Japan) or the lap protector (HAKKO Co., Nagano, Japan), which are used without anti-infective agents).

4. Recommending wound irrigation with sterile normal saline.

5. Not restricting the type of immunosuppressive agent that can be used.

6. Changing or maintaining the same gloves during the operation.

7. Changing or maintaining the surgical instruments.

Furthermore, we always maintain a normal body temperature using warming devices during surgery and do not perform preoperative hair removal.

\section{Recruitment of study participants}

The trial protocol (24 October 2018, Version 1.3) was approved by each participating institution's institutional review board and registered in the University Hospital Medical Information Network Clinical Trials Registry. Recruitment into the trial started in June 2018 and will continue until 600 participants are registered. All participants who meet the inclusion criteria will receive a participant information sheet from investigators before giving written informed consent. This study is being conducted at four general centres: Keio University Hospital (Tokyo, Japan), National Hospital Organisation Tokyo Medical Centre (Tokyo, Japan), Tokyo Saiseikai Central Hospital (Tokyo, Japan) and Kawasaki Municipal Hospital (Kanagawa, Japan).

\section{Randomisation}

Registration and allocation of participants are performed by non-blinded investigators using the CapTool Lite (Mebix, Tokyo, Japan). Eligible patients will be randomised to either surgical skin antisepsis with olanexidine (study arm A) or surgical skin antisepsis with povidone-iodine (study arm B) in a 1:1 replacement ratio. The random sequence will be generated from computer-generated block randomisation. We designated the factor of surgical approach (laparoscopy vs laparotomy) as the allocation adjustment factor because of evidence that there is a significantly higher SSI rate in laparotomy than in laparoscopy. ${ }^{20}$

\section{Blinding}

Both patients and investigators will be blinded to the assigned group. Although there is a difference in colour between povidone-iodine and olanexidine, it is feasible for patients to be masked because we wipe the stain of the antiseptic off their skin postoperatively. Non-blinded investigators cannot be masked because they will be in the operating room when the antiseptic is used.

Non-blinded investigators will answer the questionnaire about the wound condition; however, they do not diagnose the presence or absence of SSI. SSIs are diagnosed by investigators who are blinded to the group allocation with reference to the questionnaire. Blinded investigators perform data entry for diagnosis of SSI, and the data analyst is blinded.

\section{Trial visits}

Generally, patients are hospitalised 1-4 days before surgery. We obtain informed consent and record the patients' background characteristics after admission. Informed consent for the operation and clinical trial is routinely obtained on the day before surgery. Thus, randomisation is mainly performed on the day before surgery. The duration of observation will be 30 days postoperatively. The schedule for the trial visits and data collection is summarised in table 2 .

\section{Outcome measures}

The non-blinded investigators will observe the surgical wound site daily during admission. After discharge, participants will undergo outpatient observation at least

\begin{tabular}{|c|c|c|c|c|}
\hline Time point & $\begin{array}{l}\text { After } \\
\text { admission }\end{array}$ & $\begin{array}{l}\text { Before } \\
\text { surgery }\end{array}$ & Surgery & $\begin{array}{l}\text { After } \\
\text { surgery }\end{array}$ \\
\hline Informed consent & $\square$ & & & \\
\hline $\begin{array}{l}\text { Patients' } \\
\text { background } \\
\text { characteristics }\end{array}$ & $\square$ & & & \\
\hline
\end{tabular}

\begin{tabular}{l} 
Physical examination $\square$ \\
Randomisation \\
Intervention \\
$\begin{array}{l}\text { Observation of the } \\
\text { surgical site }\end{array}$ \\
\hline
\end{tabular}

口a: From postoperative day 1 to postoperative day 30 (outpatient observation is performed at least once if the discharge is within 30 days postoperatively). 
once if it is within 30 days postoperatively. Non-blinded investigators will observe the surgical wound in the same manner as during the hospital stay. We also recommend that patients visit the outpatient clinic or an emergency department if there are any symptoms suggestive of SSI such as pain or redness. If SSI is suspected based on the clinical findings, a microbiological culture would be collected using a cotton swab. The diagnosis of SSI will be determined by blinded investigators who will be unaware of the patients' group assignment. The investigator will verify the SSI via chart review using the questionnaire for SSI provided by the non-blinded investigators in accordance with the CDC guideline. Moreover, blinded investigators will assess the seriousness of all adverse events and determine whether they are related to the study.

\section{Primary outcome measure}

Postoperative 30-day SSI rate.

\section{Secondary outcome measures}

Postoperative 30-day superficial incisional SSI rate, deep incisional SSI rate, organ/space SSI rate, positive bacterial wound culture rate, bacterial strains and rates of intervention-related toxicity and allergic events (eg, erythema, pruritus, dermatitis and other symptoms of allergy around the region disinfected by the antiseptic during surgery).

\section{Definitions}

SSIs are classified as superficial incisional, deep incisional and organ/space based on criteria in the CDC guidelines (online supplementary table 1$){ }^{1}$

\section{Data collection}

All data will be collected and recorded into the web-based electric case report form (CRF; CapTool Lite) by the trial or non-blinded investigators. From the electric CRF, the trial database will be established. Patients' characteristics, such as sex, age, smoking status, body mass index, the use of prophylactic antibiotics, mode of skin closure, comorbidities, such as diabetes mellitus, and steroid use, will be collected.

Data will also be collected regarding the surgical procedures such as the type of surgery, use of laparoscopy, method of wound closure, type of prophylactic antiseptic agent, repeat application of an antiseptic agent, use of sterilised sutures for wound closure, amount of intraperitoneal irrigation, amounts of wound irrigation and blood loss and status of excision site and recorded in the electric CRF. We will confirm that personal identifying information such as patient names and medical record identification are deleted from the data. Thereafter, a linkable anonymised number is set and stored by a personal information manager for at least 5 years after study completion.

\section{Data monitoring}

Central monitoring will be conducted with the aim of ensuring that the trials are conducted safely and in accordance with the implementation plan, and the data collection is performed correctly. It is conducted once a year, with $10 \%$ of registration completed in each institution. The number of consents acquired, number of patients registered, number of patients who withdraw or are lost to follow-up and their reasons, safety, compliance with eligibility criteria and exclusion criteria, accuracy of the allocation procedure and compliance with various regulations and research plan are all evaluated by the test secretariat.

\section{Sample size calculation}

At our institution, the estimated rate of SSI after gastrointestinal surgery with wound class II is $12 \%$ (this rate was only included in a non-published Japanese report) after povidone-iodine use and $6 \%$ after olanexidine use. Assuming a group difference of $6 \%$ during the study period, 281 patients per group would provide a power of over $80 \%$, which is sufficient for detecting a difference in the proportion of SSI between olanexidine and povidone-iodine using a one-sided chi-square test at a $5 \%$ level of significance. A dropout rate of about $5 \%$ is allowed; thus, with 300 patients required per group, a total sample size of 600 patients is required for the trial.

\section{Patient and public involvement}

The patients and the public were not involved in the design of this study.

\section{Statistical analysis}

We will perform the primary analyses using the full analysis set, from which patients who do not undergo surgery or who withdraw consent before assessment of the primary endpoint are excluded. In addition, we will repeat the analyses in the per-protocol set, further excluding patients with major protocol deviations. The safety analysis set will include all patients who were randomly assigned to a study group and received treatment during the study period. For the baseline variables, summary statistics will be performed using frequencies and proportions for categorical data and means and SD for continuous variables. Patient characteristics will be compared using Pearson's $\mathrm{X}^{2}$ test or Fisher's exact test for categorical outcomes, and Student's t-test for continuous variables, as appropriate. For the primary analysis, which is aimed at comparing the treatment effects, the adjusted risk ratio and its $95 \%$ CI will be estimated using the Mantel-Haenszel method. To test for a significant association of the primary outcome, the Mantel-Haenszel test will be applied after adjusting for allocation factors. All comparisons are planned, and all $\mathrm{p}$ values will be two sided. $\mathrm{P}$ values $<0.05$ will be considered statistically significant. All statistical analyses will be performed using SAS software V.9.4. The SAP will be developed by the principal investigator and the biostatistician before completion of patient recruitment and data fixation.

\section{Ethics and dissemination}

Participant recruitment began in June 2018. The final results will be published in international peer-reviewed medical journals. 


\section{DISCUSSION}

Although some guidelines have indicated the efficacy of chlorhexidine-alcohol and povidone-iodine for reducing the SSI rate, the optimal recommendation has still not been established, and the prevalence of SSI remains high in gastrointestinal surgery. Therefore, a comparative trial between conventional antiseptics, including chlorhexidine-alcohol and povidone-iodine, and newly developed antiseptics that considers their effectiveness, toxicity and costs is needed. ${ }^{8}$

We have been conducting a randomised controlled clinical trial to compare olanexidine and povidone-iodine, which is the most popular antiseptic in Japan in terms of prevalence of SSI and its low toxicity. The strength of this trial is that we adopted blinding for diagnosing SSI at the multiple centres. To maintain the quality of practices, only four centres, all of which are high-volume centres performing more than 500 gastrointestinal surgeries per year, are participating in this trial. Furthermore, since the staff in each centre belongs to the SSI control committee, which provides unified and evidence-based counter measures against SSI at Keio University Hospital, the management of SSI at each centre can be performed in almost the same manner.

In this study, we have used povidone-iodine instead of chlorhexidine-alcohol as a control. Since chlorhexidine-alcohol is associated with inflammation, povidone-iodine is recommended and typically used for gastrointestinal surgery in Japan. In addition, chlorhexidine-alcohol at concentrations $>1 \%$ is not commercially available in Japan, although a concentration $>2 \%$ is recognised as having a bactericidal effect in international guidelines. ${ }^{21-23}$ Moreover, considering the influence of ethnic differences, including intrinsic and extrinsic ethnic factors, this comparison is a meaningful examination of SSI treatment, at least in Japan. Therefore, we think that the selection of the control group is reasonable.

Although antisepsis would influence only superficial and deep SSIs, we included organ-space SSI in the endpoint. As described earlier, this is the first study to use olanexidine; therefore, it is more important to establish evidence for all types of SSI than to limit the study to superficial and deep wound infections. Some studies have investigated skin antisepsis in gastrointestinal surgery and included organ SSI as an outcome. ${ }^{112}$

This study has several limitations. First, this trial is recruiting patients undergoing various types of gastrointestinal surgery, such as oesophagectomy, gastrectomy and cholecystectomy, which have different rates of SSI. However, there is no major bias in allocation because it is randomised. Furthermore, since this is the first report using olanexidine, it is more important to include various operations than to limit the study to a particular procedure. Second, this study is limited to the Japanese population because olanexidine is only commercially available in Japan, which could introduce an element of selection bias,

In conclusion, the present study is assessing the efficacy of olanexidine compared with povidone-iodine for preventing SSI in gastrointestinal surgery. We expect olanexidine to be more effective for preventing SSI than povidone-iodine without increasing toxicity. In the future, if superiority of olanexidine compared with povidone-iodine is proven in this trial, we should also consider conducting another trial that compares olanexidine to an alcohol-based antiseptic agent. Even if this prediction is not the final result, this trial can provide new knowledge in terms of antisepsis for preventing SSI. The result will also contribute to the development of new antisepsis treatments for gastrointestinal surgery.

\section{Trial status}

As of 25 October 2018, this trial is actively recruiting patents at three centres with additional centres planned. Two hundred of the planned 600 participants have been enrolled.

\section{Author affiliations}

${ }^{1}$ Department of Surgery, Keio University School of Medicine, Tokyo, Japan ${ }^{2}$ Department of Surgery, National Hospital Organization Tokyo Medical Center, Tokyo, Japan

${ }^{3}$ Department of Surgery, Tokyo Saiseikai Central Hospital, Tokyo, Japan ${ }^{4}$ Department of Surgery, Kawasaki Municipal Hospital, Kanagawa, Japan ${ }^{5}$ Department of Preventive Medicine and Public Health, Keio University School of Medicine, Tokyo, Japan

Acknowledgements The authors thank Asako Inoue and Kumiko Motooka, staff members of the Department of Surgery in Keio University School of Medicine, for their help in the preparation of this manuscript.

Contributors MT and $\mathrm{HO}$ contributed equally to this study. All authors made significant contribution to the conception and design of the study protocol. MT designed the study and wrote the protocol and manuscript. SM, TI, KF, RN, NW, MK, HY, YA, GO, SH, MT, TI, TY, KH, YI, YS, HH, YM, MS and TK assisted with the development of the study design and protocol. The protocol was reviewed by $\mathrm{HO}$, $\mathrm{HK}, \mathrm{MS}, \mathrm{KO}$ and YK. YS wrote the statistical analysis plan. All authors gave final approval of the manuscript and agree to be accountable for all aspects of the work.

Funding This work was supported by donations from the Department of Surgery, Keio University School of Medicine and Ohyama Health Foundation Inc.

Disclaimer Otsuka Pharmaceutical Factory Inc. was not involved in the planning of the protocol or in the conduct of the trial.

Competing interests YK received grant support from Otsuka Pharmaceutical Factory Inc.

Patient consent for publication Not required.

Ethics approval The protocol was first approved by the Institutional Review Board of Keio University School of Medicine and then approved by the institutional review board of each participating site.

Provenance and peer review Not commissioned; externally peer reviewed.

Open access This is an open access article distributed in accordance with the Creative Commons Attribution Non Commercial (CC BY-NC 4.0) license, which permits others to distribute, remix, adapt, build upon this work non-commercially, and license their derivative works on different terms, provided the original work is properly cited, appropriate credit is given, any changes made indicated, and the use is non-commercial. See: http://creativecommons.org/licenses/by-nc/4.0/.

\section{REFERENCES}

1. Berríos-Torres SI, Umscheid CA, Bratzler DW, et al. Centers for Disease Control and Prevention Guideline for the Prevention of Surgical Site Infection, 2017. JAMA Surg 2017;152:784-91.

2. Steiner HL, Strand EA. Surgical-site infection in gynecologic surgery: pathophysiology and prevention. Am J Obstet Gynecol 2017;217:121-8. 
3. López Pereira P, Díaz-Agero Pérez C, López Fresneña N, et al. 'Epidemiology of surgical site infection in a neurosurgery department'. Br J Neurosurg 2017;31:10-15.

4. Figuerola-Tejerina A, Rodríguez-Caravaca G, Bustamante-Munguira $\mathrm{J}$, et al. Epidemiological Surveillance of Surgical Site Infection and its Risk Factors in Cardiac Surgery: A Prospective Cohort Study. Rev Esp Cardiol 2016;69:842-8.

5. Maehara Y, Shirabe K, Kohnoe S, et al. Impact of intra-abdominal absorbable sutures on surgical site infection in gastrointestinal and hepato-biliary-pancreatic surgery: results of a multicenter, randomized, prospective, phase II clinical trial. Surg Today 2017;47:1060-71.

6. Kirkland KB, Briggs JP, Trivette SL, et al. The impact of surgicalsite infections in the 1990s: attributable mortality, excess length of hospitalization, and extra costs. Infect Control Hosp Epidemiol 1999;20:725-30.

7. de Lissovoy G, Fraeman K, Hutchins V, et al. Surgical site infection: incidence and impact on hospital utilization and treatment costs. Am $J$ Infect Control 2009;37:387-97.

8. Allegranzi B, Bischoff P, de Jonge S, et al. New WHO recommendations on preoperative measures for surgical site infection prevention: an evidence-based global perspective. Lancet Infect Dis 2016:16:e276-e287.

9. Srinivas A, Kaman L, Raj P, et al. Comparison of the efficacy of chlorhexidine gluconate versus povidone iodine as preoperative skin preparation for the prevention of surgical site infections in clean-contaminated upper abdominal surgeries. Surg Today 2015;45:1378-84.

10. Springel EH, Wang XY, Sarfoh VM, et al. A randomized openlabel controlled trial of chlorhexidine-alcohol vs povidone-iodine for cesarean antisepsis: the CAPICA trial. Am J Obstet Gynecol 2017;217:463.e1-463.e8.

11. Darouiche RO, Wall MJ, Itani KM, et al. Chlorhexidine-Alcohol versus Povidone-lodine for Surgical-Site Antisepsis. N Engl J Med 2010;362:18-26.

12. Park HM, Han SS, Lee EC, et al. Randomized clinical trial of preoperative skin antisepsis with chlorhexidine gluconate or povidone-iodine. Br J Surg 2017;104:e145-e150.
13. National Institute for Health and Clinical Excellence (NICE). Surgical Site 3 Infection: Prevention and Treatment of Surgical Site Infection (NICE Clinical Guideline 74), 2008.

14. Burlingame BDB, Link T. Guideline for preoperative patient skin antisepsis. Connor R, ed. AORN guidelines for perioperative practice. Denver, CO: Association of periOperative Registered, Nurses, 2016.

15. Zamora JL, Price MF, Chuang P, et al. Inhibition of povidone-iodine's bactericidal activity by common organic substances: an experimental study. Surgery 1985;98:25-9.

16. Lee I, Agarwal RK, Lee BY, et al. Systematic review and cost analysis comparing use of chlorhexidine with use of iodine for preoperative skin antisepsis to prevent surgical site infection. Infect Control Hosp Epidemiol 2010;31:1219-29.

17. Lim KS, Kam PC. Chlorhexidine--pharmacology and clinical applications. Anaesth Intensive Care 2008;36:502-12.

18. Inoue $\mathrm{Y}$, Hagi A, Nii T, et al. Novel antiseptic compound OPB-2045G shows potent bactericidal activity against methicillin-resistant Staphylococcus aureus and vancomycin-resistant Enterococcus both in vitro and in vivo: a pilot study in animals. J Med Microbiol 2015;64:32-6.

19. Hagi A, Iwata K, Nii T, et al. Bactericidal Effects and Mechanism of Action of Olanexidine Gluconate, a New Antiseptic. Antimicrob Agents Chemother 2015;59:4551-9.

20. Howard DP, Datta G, Cunnick G, et al. Surgical site infection rate is lower in laparoscopic than open colorectal surgery. Colorectal Dis 2010;12:423-7.

21. Preventing surgical site infections. Key recommendations for practice. Dublin: Joint Royal College of Surgeons in Ireland/Royal Colleges of Physicians of Ireland Working Group on Prevention of Surgical Site Infection 2012.

22. Targeted literature review: What are the key infection prevention and control 13 recommendations to inform a surgical site infection (SSI) prevention quality improvement 14 tool? Version 3.0. February 2015. Edinburgh: Health Protection Scotland, 2015.

23. High impact intervention: care bundle to prevent surgical site infection. London: Department of Health, 2011. 